\title{
Free Random Lévy Variables and Financial Probabilities
}

\author{
Zdzisław Burda ${ }^{\mathrm{a}, \mathrm{b}}$, Jerzy Jurkiewicz ${ }^{\mathrm{a}}$, Maciej A. Nowak ${ }^{\mathrm{a}, 1}$, \\ Gábor Papp ${ }^{\mathrm{c}}$, Ismail Zahed ${ }^{\mathrm{d}}$ \\ ${ }^{a}$ M. Smoluchowski Institute of Physics, Jagellonian University, Cracow, Poland \\ ${ }^{\mathrm{b}}$ Fakultät für Physik, Universität Bielefeld P.O.Box 100131, D-33501 Bielefeld, \\ Germany \\ ${ }^{\mathrm{c}} H A S$ Research Group for Theoretical Physics, Eötvös University, Budapest, \\ H-1518 Hungary \\ ${ }^{\mathrm{d}}$ Department of Physics and Astronomy, SUNY-Stony-Brook, NY 11794 U.S.A.
}

\begin{abstract}
We suggest that Free Random Variables, represented here by large random matrices with spectral Lévy disorder, may be relevant for several problems related to the modeling of financial systems. In particular, we consider a financial covariance matrix composed of asymmetric and free random Lévy matrices. We derive an algebraic equation for the resolvent and solve it to extract the spectral density. The free eigenvalue spectrum is in remarkable agreement with the one obtained from the covariance matrix of the SP500 financial market.
\end{abstract}

Noise with power law distribution is encountered in numerous stochastic systems in physics, biophysics and finances (e.g. financial time series) $[1,2]$. One of the crucial problems in such systems is an understanding of the character of correlations between numerous agents. It is therefore tempting to try to find a description, in which we could follow the stochastic evolution not of a single object, but rather an evolution of an array (matrix) built of many objects. Such description requires basically a generalization of the classical concepts of theory of probability to non-commuting variables. This fundamental concept has been introduced by Voiculescu, using the powerful theory of free random variables [3]. Another major twist happened when it was realized, that abstract non-commuting operators representing FRV have an explicit realization in terms of certain large random matrices, when the size of the matrix

1 Talk at NATO Advanced Research Workshop "Applications of Physics to Economic Modeling", Prague, 8-10 February, 2001.

Preprint submitted to Elsevier Preprint 31 October 2018 
tends to infinity. Free random matrices with Gaussian fixed point, have been applied to many physical problems [4-6]. Recently, using some of the concepts developed in [3], we extended the concept of free random Lévy variables to matrices [7], and suggested that the results may be relevant for addressing the issue of noise in stochastic systems with power law distributions. In this talk, we explain in a pedagogical way what is the corner-stone of stability for noncommuting variables. Then we move towards a sample application, discussing the financial correlation matrix.

Let us consider a Gaussian distribution in classical theory of probability, i.e. the distribution

$$
p(x)=\frac{1}{\sqrt{2 \pi} \sigma} e^{-\frac{x^{2}}{2 \sigma^{2}}}
$$

with the variance $\sigma$. Now let us ask the question, what is the distribution of the sum of two identical independent Gaussian distributions, i.e. what is the distribution of

$$
S_{2}=x_{1}+x_{2}
$$

where $P_{2}\left(S_{2}\right)=p\left(x_{1}\right) \otimes p\left(x_{2}\right)$. Since the Fourier transform unwinds the convolution, if we introduce the characteristic functions

$$
\begin{aligned}
& \phi(q)=\int p(x) e^{i q x} d x \\
& p(x)=\frac{1}{2 \pi} \int \phi(q) e^{-i q x} d q,
\end{aligned}
$$

then, $\Phi_{2}(q)=\phi(q) \cdot \phi(q)$. The inverse Fourier transform gives

$$
P_{2}\left(S_{2}\right)=\frac{1}{\sqrt{2 \pi} \sqrt{2} \sigma} e^{-\frac{x^{2}}{2(\sqrt{2} \sigma)^{2}}}
$$

i.e. again the normal distribution but with the new variance $\sigma_{2}=\sqrt{2} \sigma$. One may say, that the logarithm of the characteristic function

$$
\ln \Phi_{2}(q)=\ln \phi(q)+\ln \phi(q)
$$

realizes a certain additivity law for Gaussian variables (2).

Is it possible to find an analog of this construction for random matrix ensembles? Consider again a Gaussian ensemble built of large $N \times N$ matrices $M$, for which the pertinent resolvent is generically given by 


$$
G(z)=\int d M e^{-N V(M)} \frac{1}{N} \operatorname{Tr}\left(\frac{1}{z-M}\right)
$$

where the the potential $V(M)$ equals $\frac{1}{2} \operatorname{Tr} M^{2}$. For simplicity we put the scale of the Gaussian distribution equal to one. This Green's function fulfills a simple algebraic equation $G(z)=(z-G(z))^{-1}$, and the normalizable solution reads

$$
G(z)=\frac{1}{2}\left(z-\sqrt{z^{2}-4}\right)
$$

Using the fact, that the averaged spectral distribution is related to the discontinuities of the Green's function ${ }^{2}$

$$
\rho(\lambda)=-\left.\frac{1}{\pi} \lim _{\epsilon \rightarrow \infty} \operatorname{Im} G(z)\right|_{z=\lambda+i \epsilon}
$$

we recover Wigner's semicircle law

$$
\rho(\lambda)=\frac{1}{2 \pi} \sqrt{4-\lambda^{2}}
$$

Let us now try to add two independent matrix Gaussian ensembles, as we did in the case of commuting variables of the classical probability theory. In other words, we are asking what is the distribution of the eigenvalues, if the corresponding Green's function is given by

$$
\begin{aligned}
G_{1+2}(z)= & \int d M_{1} d M_{2} e^{-N V_{1}\left(M_{1}\right)} e^{-N V_{2}\left(M_{2}\right)} \\
& \frac{1}{N} \operatorname{Tr}\left(\frac{1}{z-\left(M_{1}+M_{2}\right)}\right) .
\end{aligned}
$$

This time the "convolution" is matrix-valued, and the matrices $M_{1}, M_{2}$ do not commute!

Free Random Variable (FRV) calculus provides a surprisingly simple solution to this problem. First, let us find the functional inverse of the Green's functions for ensembles $M_{1}$ and $M_{2}$, i.e. find the solution $G[B(z)]=z$ for every $z$ for both ensembles. Then, define $R(z)=B(z)-1 / z$ for both ensembles. The function $\mathrm{R}$ is additive

$$
R_{1+2}(z)=R_{1}(z)+R_{2}(z)
$$

$\overline{2}$ It is obvious using special functions property $\frac{1}{x+i \epsilon}=P V \frac{1}{x}-i \pi \delta(x)$. 
Proceeding now in the reverse order one can reconstruct $G_{1+2}(z)$.

We exemplify this construction considering two identical Gaussian ensembles (6). Since for both the Green's function is given by (7), we get

$$
B_{1}(z)=B_{2}(z)=z+1 / z
$$

so $R_{1}(z)=R_{2}(z)=z$ and the additivity law gives

$$
R_{1+2}(z)=R_{1}(z)+R_{2}(z)=z+z=2 z
$$

or, equivalently, $B_{1+2}(z)=2 z+1 / z$. By substitution $z \rightarrow G_{1+2}(z)$ and by the definition of the functional inverse, we immediately get

$$
G_{1+2}(z)=\frac{1}{4}\left(z-\sqrt{z^{2}-8}\right)
$$

The discontinuity of this function yields the spectral distribution

$$
\rho_{1+2}(\lambda)=\frac{1}{4 \pi} \sqrt{8-\lambda^{2}}
$$

The matrix convolution of two Gaussian ensembles is again a Gaussian ensemble, but rescaled by a factor $\sqrt{2}$.

The additivity law (11) in non-commutative probability theory of random matrices forms an analog of the additivity law for the logarithms of the characteristic functions in case of classical probability theory.

This example demonstrates that the techniques of FRV offer a powerful shortcut when we seek the distribution of variables coming from "sum" of different ensembles. The above construction could be generalized to higher-order polynomial measures, sums of random and deterministic ensembles, free products of ensembles and also to strictly non-hermitian ensembles $[5,6]$.

We focus now on the crucial question of stability. We know that in the classical probability theory the concept of Gaussian stability (central limit theorem) was generalized by Lévy and Khintchine to distributions with a power-law tail. Is it possible that the Gaussian stability for FRV allows for a similar generalization? Works of Bercovici and Voiculescu [3] provide a positive, but formal answer to this problem. Recently, we have suggested an explicit construction for the matrix measure of such ensembles, borrowing on the Coulomb gas analogy well-known in random matrix theory. Generically, the potential is given by 


$$
V^{\prime}(\lambda)=2 \operatorname{Re} G(\lambda+i 0)
$$

and for free Lévy matrices, $G(z)$ satisfies an algebraic equation in large $N$ limit $[7](\alpha \neq 1)$

$$
b G^{\alpha}(z)-(z-a) G(z)+1=0
$$

in the upper half-plane, and follows by Cauchy reflection in the lower halfplane. The parameter $b$ is related to the Lévy index $\alpha$, asymmetry $\beta$, and range or scale $\gamma$. Further details are discussed in [7]. We would like to mention, that the explicit form of the matrix measure is known only in few cases. Even in these cases, Lévy ensembles exhibit very nontrivial behavior, and the potentials are non-analytic, contrary to the broadly studied polynomial cases.

Let us apply some of these observations to econophysics. Recently, it was pointed out that financial covariances are permeated by Gaussian noise in the low-lying eigenvalue region with consequences on risk assessment [8]. Here we show, following our recent analysis [9], that the financial covariance spectrum is throughout permeated by free Lévy noise. We quantify the fluctuations in a stochastic system by the use of a covariance matrix $\mathbf{C}$,

$$
\mathbf{C}_{i j}=\frac{1}{T^{2 / \alpha}} \sum_{t=1}^{T} M_{t i} M_{t j}
$$

where $M_{t i}$ is $T \times N$ (asymmetric). The normalization in (18) follows from the fact that the "variance" for Lévy matrices grows like $T^{2 / \alpha}$ where $\alpha=2$ is the expected diffusive limit (Brownian). Free Lévy ensembles are super-diffusive for $0<\alpha<1$ and sub-diffusive for $1<\alpha<2$ with $\alpha=1$ the critical divide. We define the empirical matrix of relative returns

$$
M_{t i}=\left(x_{i t+1}-x_{i t}\right) / x_{i 0}-\langle M\rangle
$$

where $x_{i t}$ are the raw returns made of the price $x_{i t}$ of stock $\mathrm{i}$ at time t. The returns are normalized to the initial price $x_{i 0}$ to insure scale invariance. They carry zero mean after subtracting the average relative return $\left\langle M_{t i}\right\rangle_{t, i}$. For the raw prices we will use the daily quotations of $N=406$ stocks from the SP500 market over the period of $T+1=1309$ days from 01.01.1991 till 06.03.1996 (ignoring dividends). For these data the matrix asymmetry is $m=1308 / 406 \approx$ 3.22 .

The spectrum of $\mathbf{C}$ contains important information about the character of the correlations. Using Voiculescu's powerful machinery of $R$ and $S$ transforms for free random variables [3] and some of the techniques developed in our 

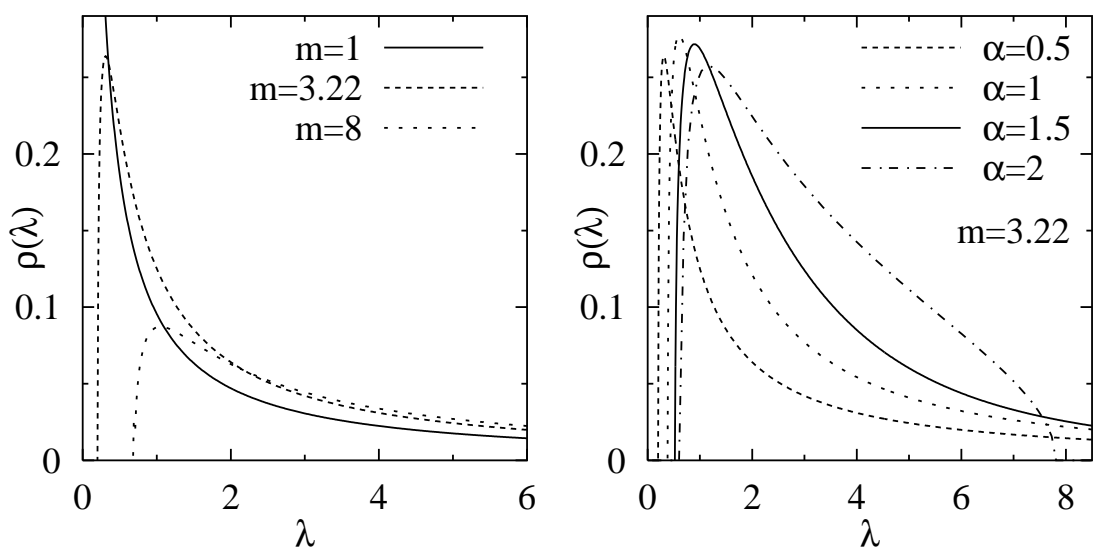

Fig. 1. Left: Spectral density of FRV with $\alpha=1 / 2$ and different asymmetry parameters $m$. Right: spectral density for several indices $\alpha$, at $m=3.22$.

earlier papers we find that the resolvent for the covariance built from pure Lévy matrices $M$ satisfies the transcendental equation $(\gamma=1)[9]$

$$
G(z)=\frac{1+w(z)}{z}
$$

where $w(z)$ satisfies the multi-valued equation

$$
-e^{i \frac{2 \pi}{\alpha}} \cdot w^{2 / \alpha} \cdot z=(1+w)(w+m)
$$

for fixed asymmetry $m=T / N$ and Lévy asymmetry parameter $\beta=0$. The distribution of eigenvalues of the covariance matrix follows from the discontinuity of $G(z)$. The distribution is unimodal and it's support extends to infinity. Since the covariance is a square, the tail distribution is characterized by an index $\alpha / 2$ since the entries $M_{t i}$, are Lévy distributed with index $\alpha$.

In Fig. 1 we show some typical spectra. The case $\alpha=2$ corresponds to Gaussian distributions used recently in [8].

In Fig. 2 we compare the analytical results following from the free Lévy covariance to the ones following from the SP500 market. Fig. 2 shows the distribution of eigenvalues for free Lévy matrices with index $\alpha / 2=3 / 4$ and asymmetry $m=2$, versus the raw SP500 data (left) and the reshuffled SP500 data (right). The reshuffled data are obtained by randomly permuting the time ordering of the price series, independently for all stocks, destroying all inter-stock correlations. Our optimal fit preserves the index of the cumulative distribution, albeit for a smaller asymmetry $(m=2<3.22)$. It is remarkable that our free Lévy fit to the spectrum of the covariance suggests that the time series of returns is power law distributed with index $\alpha=3 / 2$ which is consistent with detailed studies of the SP500 financial time series [2]. 


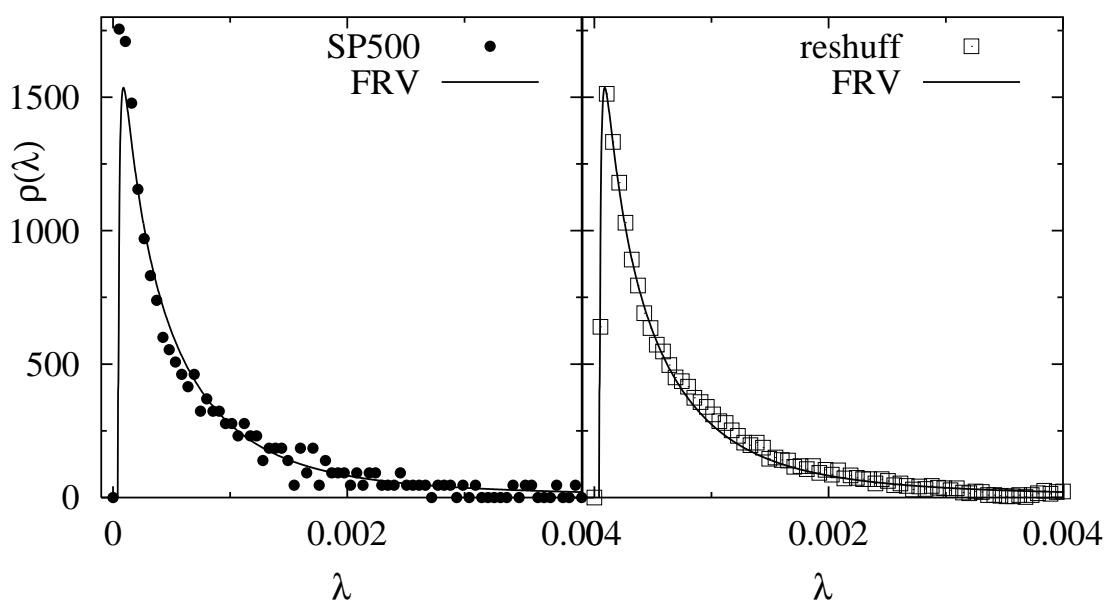

Fig. 2. Raw (left) and reshuffled (right) SP500 spectrum compared to the FRV result with $m=2$.

In this talk, we have summarized some of our recent results on free random Lévy matrices and finances [7,9]. A more thorough analysis and discussions including (non-rotationally invariant) ensembles of Lévy matrices [10] can be found in $[7,9]$. We have shown that the spectral density of the SP500 financial covariance is in overall agreement with a free Lévy distribution of index $\alpha / 2=$ $3 / 4$. Free Lévy noise may be dominant in financial covariances, a point that may affect all appraisals of risk in finances. In general, we expect the results discussed in this talk to be of relevance to a number of problems involving non-commutative probabilities, whether in physics, biophysics, social sciences or finances.

\section{Acknowledgments:}

MAN thanks the organizers of the Workshop for their hospitality. This work was supported in part by the US DOE grant DE-FG02-88ER40388, by the Polish Government Project (KBN) 2P03B 01917, by the Hungarian Ministry of Education FKFP grant 220/2000 and by EC IHP grant HPRN-CT-199900161.

\section{References}

[1] R. Mantegna and H. Stanley, An Introduction to Econophysics, Cambridge Univ. (2000).

[2] J. Bouchaud and M. Potters, Theory of Financial Risks, Cambridge Univ. (2000).

[3] D.V. Voiculescu, Invent. Math. 104 (1991) 201; D.V. Voiculescu, K.J. Dykema and A. Nica, "Free Random Variables", Am. Math. Soc., Providence, RI (1992); 
H. Bercovici and D. Voiculescu, Ind. Univ. Math. J.42 (1993) 733.

[4] R. Gopakumar and D. Gross, e-print hep-th/9411021

[5] A. Zee, Nucl. Phys. B474 )1996) 726; J. Feinberg and A. Zee, Nucl. Phys. B501 (1997) 643; ibid. B504 (1997) 579.

[6] R.A. Janik, M.A. Nowak, G. Papp and I. Zahed, Acta Phys. Pol. B28 (1997) 2949; e-print hep-th/9710103; R.A. Janik, M.A. Nowak, G. Papp, J. Wambach and I. Zahed, Phys. Rev. E55 (1997) 4100; R.A. Janik, M.A. Nowak, G. Papp and I. Zahed, Nucl. Phys. B501 (1997) 603.

[7] Z. Burda, R. Janik, J. Jurkiewicz, M.A. Nowak, G. Papp and I. Zahed, Free random Lèvy Matrices, e-print cond-mat/0011451.

[8] L. Laloux, P. Cizeau, J. Bouchaud and M. Potters, Phys. Rev. Lett. 83 (1999) 1467, e-print cond-mat/9810255; V. Plerou, P. Gopikrishnan, B. Rosenow, L. Nunes Amaral, and H. Stanley, Phys. Rev. Lett. 83 (1999) 1471, e-print cond-mat/9902283.

[9] Z. Burda, J. Jurkiewicz, M.A. Nowak, G. Papp and I. Zahed, Lévy Matrices and Financial Covariances, e-print cond-mat/0103108, Free Lévy matrices and Financial Correlations, e-print cond-mat/0103109.

[10] P. Cizeau and J.-P. Bouchaud, Phys. Rev. E50 (1994) 1810. 